\title{
Identification of a Golgi-localized UDP-N- acetylglucosamine transporter in Trypanosoma cruzi
}

\author{
Carlos Gustavo Baptista ${ }^{1 \dagger}$, Elizabeth Cristina Rodrigues ${ }^{2 \dagger}$, Patricia Morking $^{3}$, Amanda Klinke ${ }^{3}$, Maria Luiza Zardo ${ }^{3}$, \\ Maurílio José Soares ${ }^{3}$, Alessandra Melo de Aguiar ${ }^{3}$, Samuel Goldenberg ${ }^{3}$ and Augusto Savio Peixoto Ramos ${ }^{3^{*}}$
}

\begin{abstract}
Background: Nucleotide sugar transporters (NSTs) play an essential role in translocating nucleotide sugars into the lumen of the endoplasmic reticulum and Golgi apparatus to be used as substrates in glycosylation reactions. This intracellular transport is an essential step in the biosynthesis of glycoconjugates.

Results: We have identified a family of 11 putative NSTs in Trypanosoma cruzi, the etiological agent of Chagas' disease. A UDP-N-acetylglucosamine transporter, TcNST1, was identified by a yeast complementation approach. Based on a phylogenetic analysis four candidate genes were selected and used for complementation assays in a Kluyveromyces lactis mutant strain. The transporter is likely expressed in all stages of the parasite life cycle and during differentiation of epimastigotes to infective metacyclics. Immunofluorescence analyses of a GFP-TcNST1 fusion protein indicate that the transporter is localized to the Golgi apparatus. As many NSTs are multisubstrate transporters, we also tested the capacity of TCNST1 to transport GDP-Man.

Conclusions: We have identified a UDP-N-acetylglucosamine transporter in T. cruzi, which is specifically localized to the Golgi apparatus and seems to be expressed, at the mRNA level, throughout the parasite life cycle. Functional studies of TCNST1 will be important to unravel the role of NSTs and specific glycoconjugates in T. cruzi survival and infectivity.
\end{abstract}

Keywords: Glycoconjugates, Nucleotide sugar, Transporter, Trypanosomatids

\section{Background}

Trypanosoma cruzi is the etiological agent of Chagas' disease, which affects approximately 7 million people, mostly in Latin America, but also in other regions due to migration and blood transfusion [1]. The life cycle of this parasite is complex, involving at least four well-defined life forms: the proliferative forms present in both the insect vector (epimastigotes) and mammals (intracellular amastigotes) and the infectious non-dividing metacyclics (insect stage) and bloodstream trypomastigotes [2].

The dense glycocalyx of $T$. cruzi plays a fundamental role in survival and infectivity, and its molecular composition depends on the parasite's life form. The glycocalyx is rich in glycoinositolphospholipids (GIPLs), either

\footnotetext{
* Correspondence: aspramos@fiocruz.br

${ }^{\dagger}$ Equal contributors

${ }^{3}$ Instituto Carlos Chagas, Fiocruz Paraná, Curitiba 81350-010, PR, Brazil

Full list of author information is available at the end of the article
}

free or as protein-membrane anchors. Free GIPLs are major cell surface constituents of the insect stages of the parasite acting as modulators of the host immune system [3] and in epimastigote attachment to the midgut surface of the vector [4].

Mucins, the most abundant glycoproteins of T. cruzi, are highly O-glycosylated proteins tethered to the membrane via glycosylphosphatidylinositol (GPI) anchors. They are involved in protection against intestinal proteases in the insect vector [5] and with the attachment to and invasion of mammalian cells [6]. In terms of structure, it is important to note that the O-linked glycans of T. cruzi mucins are attached to serine (Ser) or threonine (Thr) residues by $\mathrm{N}$-acetylglucosamine instead of $\mathrm{N}$-acetylgalactosamine as it occurs in most mammalian mucins [7].

As in other eukaryotes, the synthesis of T. cruzi glycoconjugates occurs in the lumen of the endoplasmic reticulum (ER) and Golgi apparatus through the action of 
glycosyltransferases using nucleotide sugars as substrates. These sugar-activated donors must be transported across the ER and Golgi membranes by nucleotide sugar transporters (NSTs). This intracellular transport is essential for proper protein and lipid glycosylation.

NSTs comprise a family of structurally related and highly hydrophobic type III transmembrane proteins, which have been studied in different organisms, from yeast to human [8]. Based on a detailed membrane topology study of the mouse CMP-sialic acid transporter [9], these transporters are supposed to have 10 transmembrane (TM) domains with both amino- and carboxyl-termini facing the cytosol. Mutations in NSTs are associated with widespread defects in glycosylation leading to developmental diseases in mammals and loss or attenuated infectivity of human pathogens [10].

In parasitic protozoa, the role of NSTs has been investigated in Leishmania spp. [11, 12], T. brucei [13] and Toxoplasma gondi [14]. In L. major, knockout of the GDPmannose (GDP-Man) transporter LPG2 resulted in mutants deficient in cell-surface phosphoglycan molecules displaying attenuated virulence in mice [15]. Recently, four NSTs from T. brucei (TbNST1-4) were characterized [13]. Silencing and knockout experiments of TbNST4, which transports UDP- $N$-acetylglucosamine (UDP-GlcNAc), UDP$\mathrm{N}$-acetylgalactosamine (UDP-GalNAc) and GDP-Man, resulted in glycosylation defects, but no impairment of infectivity was observed, likely due to redundancy in function.

In this study, we have identified a UDP-GlcNAc transporter from T. cruzi (named TcNST1) by yeast complementation in vivo. We show that TcNST1 is localized to the Golgi apparatus and that the gene is likely expressed during the parasite life cycle and in vitro metacyclogenesis-a process by which epimastigote forms differentiate into infective metacyclic trypomastigotes. This is the first experimentally characterized NST in T. cruzi, for which there is relatively little information regarding glycoconjugate biosynthesis.

\section{Results}

\section{Trypanosoma cruzi NST candidates}

We initially searched for putative nucleotide sugar transporters in the T. cruzi genome by performing Blastp searches in GeneDB [16] using characterized NSTs of different organisms as queries. We have identified a family of eleven putative NSTs (Table 1) showing considerable similarity (e-value $<1 \mathrm{e}-10$ ) to known NSTs. Consistent with their putative assigned function, the genes code for multi-transmembrane (TM) proteins displaying between 7 and 10 TM domains (Table 1).

Due to the hybrid nature of the diploid CL Brener strain [17], both haplotypes of nine of the genes could be identified based on similarity and synteny (alleles are indicated in parentheses in Table 1). The alleles in each pair are at least $90 \%$ identical to each other at the amino acid level and, with two exceptions, code for proteins of identical or similar size (up to three amino acids in difference). The genes TcCLB.509127.90 and TcCLB.511817.280, however, have an extended amino terminus when compared to their respective alleles. Comparison of the predicted protein products with the corresponding homologues in $T$. cruzi Sylvio $\times 10 / 1$, a non-hybrid strain whose draft genome sequence was made available more recently [18], and also with the putative homologues in T. brucei and Leishmania spp., suggests that for both genes the amino terminal extension results from annotation errors (data not shown).

Table 1 Putative NSTs identified in the T. cruzi genome (Clone CL Brener)

\begin{tabular}{llll}
\hline Gene $^{\mathrm{a}}$ & Number of amino acids & $\begin{array}{l}\text { Identity in amino acids } \\
\text { between alleles (\%) }\end{array}$ & TM domains $^{\mathrm{b}}$ \\
\hline TCCLB.511517.150 (TcCLB.511737.70) (TcNST1) & $313(313)$ & 99 & 9 \\
TCCLB.511301.50 (TcCLB.511353.30) & $316(316)$ & 98 & 8 \\
TCCLB.510355.220 (TcCLB.506753.60) & $322(322)$ & 97 & 9 \\
TCCLB.506509.40 (TcCLB.506579.80) & $347(344)$ & 95 & 9 \\
TCCLB.509741.20 (TCCLB.507625.200) & $355(355)$ & 96 & 10 \\
TCCLB.506793.40 (TCCLB.510611.20) & $387(390)$ & 90 & 8 \\
TCCLB.504085.60 (TCCLB.507089.40) & $412(412)$ & 93 & 9 \\
TCCLB.508737.180 (TCCLB.509127.90) & $444(494)$ & 96 & 9 \\
TCCLB.511817.280 (TCCLB.510531.10) & $541(484)$ & 98 & 7 \\
TCCLB.511277.400 & 350 & - & 9 \\
TCCLB.504057.120 & 358 & - & 9 \\
\hline
\end{tabular}

${ }^{a}$ Eleven putative T. cruzi NSTs were identified in GeneDB by perfoming Blastp searches using characterized NSTs of different organisms as queries. For nine of the genes both haplotypes could be identified based on similarity and synteny. The hybrid diploid CL Brener strain is composed of haplotypes named Esmeraldo-like and non-Esmeraldo-like. Genes (and their respective number of amino acids) derived from Esmeraldo-like haplotypes are indicated in parentheses

${ }^{\mathrm{b}}$ Number of transmembrane domains (for non-Esmeraldo like genes only) were predicted using the TMHMM Server 2.0 (http://www.cbs.dtu.dk/services/TMHMM/) 


\section{T. cruzi UDP-GIcNAc transporter}

It is not possible to determine the substrate specificity of a given NST by analysis of its amino acid sequence [8]. To identify UDP-GlcNAc transporters, we used a yeast complementation approach in which a Kluyveromyces lactis mutant deficient in the Golgi transport of UDPGlcNAc was used [19]. This mutant has been used for the identification of UDP-GlcNAc transporters from canine cells [20], Caenorhabditis elegans [21] and T. brucei [13]. Complementation assays are based on the less intense binding of the GS-II lectin from Griffonia simplicifolia, which specifically recognizes terminal GlcNAc residues, to the mutant cell surface.

Although sequence similarity is not particularly useful for substrate specificity prediction, we used the UDP-GlcNAc transporters from T. brucei [13], a closely related parasite, to restrict our candidate genes. Based on phylogenetic analysis between the putative $T$. cruzi transporters and known NSTs from different organisms (Fig. 1), the genes TcCLB.511277.400, TcCLB.504085.60, TcCLB.504057.120 and TCCLB.511517.150 code for the closest transporters to TbNST1, 2, 3 and 4, respectively. These genes, indicated by arrows in Fig. 1, were then used for the complementation assays in K. lactis.

Only the transporter encoded by the TcCLB.511517.150 gene, TcNST1, could rescue the wild-type phenotype (Fig. 2a). Restoration of GSII lectin binding to levels similar to those of wild-type cells was observed in approximately $50 \%$ of cells transfected with TcNST1. Similar results have been observed for the heterologous expression of NSTs in yeast, and it is likely related to the expression of nonfunctional transporters [22]. The mean fluorescence intensity values are presented in Fig. $2 \mathrm{~b}$. For cells expressing TcNST1, the fluorescence intensity corresponded to approximately $60 \%$ of wild-type levels, a finding that is in agreement with half of the cells not restoring GS-II lectin binding. As a positive control, we used the K. lactis UDP-GlcNAc transporter (Kl UGT), whose activity is deficient in the yeast mutant strain. The expression of the other genes in the $K$. lactis mutant cells did not increase binding of the lectin (Additional file 1). Expression of these genes in $K$. lactis was analyzed by western blotting using a monoclonal antibody against the histidine tail inserted at the carboxyl-terminal of the transporters. However, our results regarding level of expression and apparent molecular weight were not conclusive (data not shown). Therefore, the incapacity of TCCLB60, TcCLB120 and TCCLB400 to complement the $K$. lactis mutant could be due to lack of proper expression in the yeast cells.

The TcCLB.511517.150 gene and its allele TcCLB.511737.70 are both composed of 942 nucleotides and code for a 313-amino acid polypeptide. There is only one amino acid difference between them, in which an asparagine residue is replaced by a serine (N74S) in TcCLB.511517.150.
Sequencing of the gene from T. cruzi clone Dm28c, which was used in this work, revealed a protein identical to its homologue in T. cruzi Sylvio $\times 10 / 1$, encoded by the gene TCSYLVIO_003299. This protein differ by three amino acids to that encoded by the gene TcCLB.511517.150 in T. cruzi CL Brener (K7N, L168V, and L234F in TcCLB.511517.150).

Many NSTs are multisubstrate transporters, including the recently characterized $T$. brucei transporters. TbNST4 is the closest T. brucei transporter to TcNST1, and it can transport UDP-GlcNAc, UDP-GalNAc and GDP-Man. Next, we decided to test whether TcNST1 would also be able to transport GDP-Man because mannose residues are important for T. cruzi glycoconjugates (GalNAc residues are not present in either T. cruzi or T. brucei glycoconjugates). The capacity to transport GDP-Man was assessed by complementation of a Saccharomyces cerevisiae mutant partially deficient in GDP-Man transport (NDY5 strain) [23] using a fusion protein TcNST1-V5. As shown in Fig. 3a, TcNST1 could not restore growth of the yeast cells in the presence of the anionic dye Congo red. To rule out the possibility that TcNST1 was not expressed in the yeast cells, western blot analyses were carried out using an anti-V5 monoclonal antibody. TcNST1 is visualized in total protein extracts of cells transfected with the vector encoding TcNST1-V5 but not in cells transfected with the empty vector (Fig. 3b).

\section{TcNST1 subcellular localization and gene expression}

That TcNST1 could restore UDP-GlcNAc transport in the $K$. lactis mutant suggested the Golgi apparatus as its probable subcellular localization. To investigate this hypothesis in $T$. cruzi, we used a chimeric protein in which green fluorescent protein (GFP) was fused to the $\mathrm{N}$-terminus of TcNST1. After transfection and selection of neomycinresistant cells, $T$. cruzi epimastigotes were analyzed by fluorescence microscope. Transfected epimastigotes with the GFP-TcNST1 fusion protein displayed a dot signal at the anterior region of the parasite while fluorescence emitted by transfectants expressing only GFP-Flag (GFP fused at the C-terminus with Flag) was distributed through the cytoplasm. Wild-type cells had little intrinsic fluorescence (Additional file 2). Next we used a monoclonal antibody against GFP to detect expression of GFP-TcNST1 in total extracts of epimastigotes. As shown in Additional file 3, the transporter fused to GFP is present in transfected epimastigotes but not in wild-type cells. The Golgi localization of TcNST1 was confirmed by co-localization with TcHIP, a putative $T$. cruzi zDHHC palmitoyl transferase recently shown to be localized to the Golgi apparatus [24]. A clear punctual signal in close contact with the kinetoplast, as would be expected for a Golgi resident protein (Fig. 4b, d), was shown to co-localize with TcHIP (Fig. 4c, d). 




TcNST1 expression was analyzed by semi-quantitative RT-PCR along the life cycle of T. cruzi and during the process of metacyclogenesis in vitro, in which epimastigotes in the late exponential phase are differentiated into metacyclics under nutritional stress in a chemically defined differentiation medium [25]. In this medium, called TAU3AAG, epimastigotes adhere to a substrate and are released as metacyclics after approximately $100 \mathrm{~h}$. 


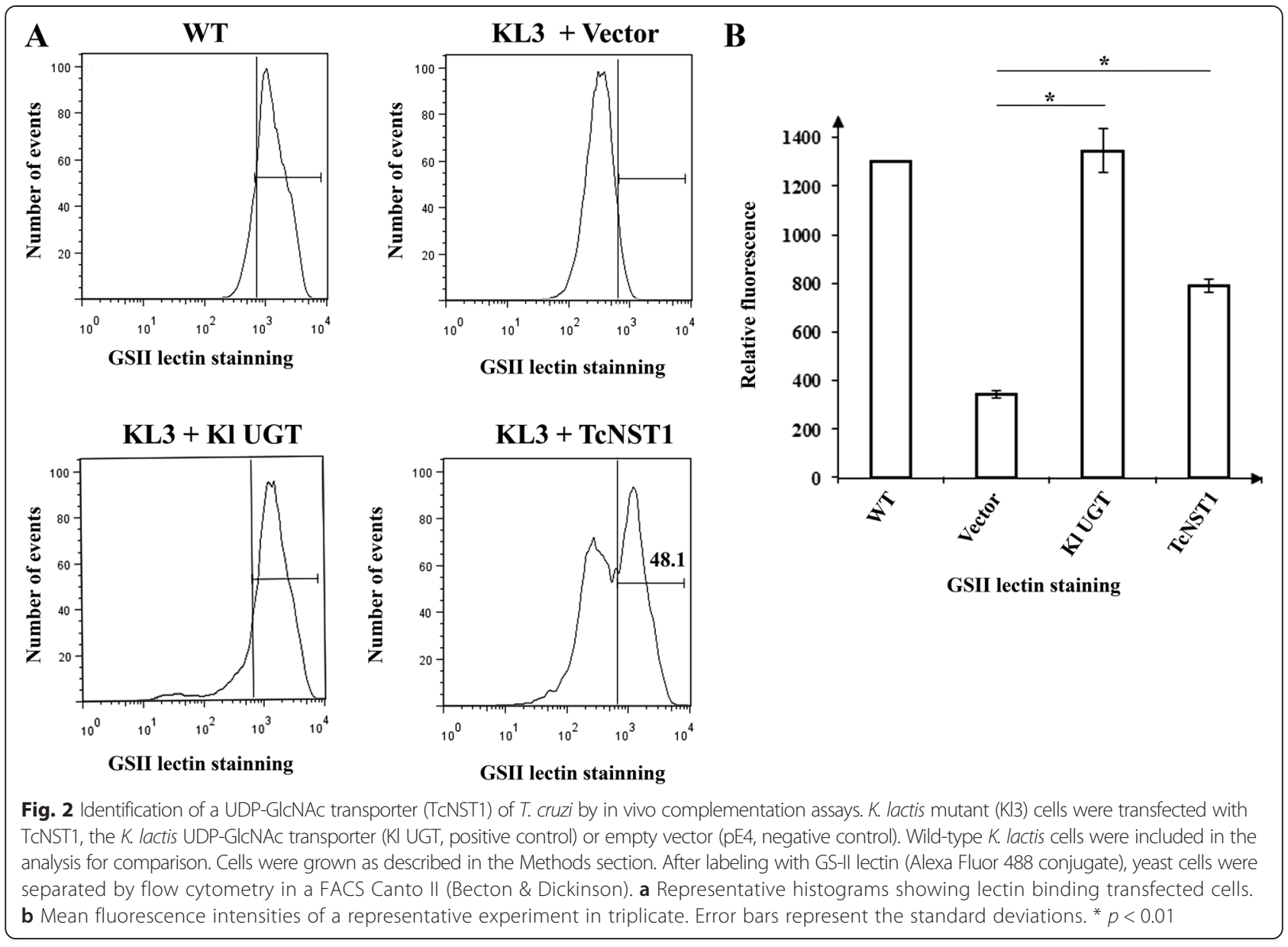

According to the results shown in Fig. 5, TcNST1 mRNA is present in all four major life forms of T. cruzi and in intermediate forms during differentiation from epimastigotes to metacyclics. While post-transcriptional control of gene expression is particularly important in trypanosomatids, the presence of mRNA suggests that the transporter may be expressed in all life forms of the parasite.

\section{Discussion}

We have identified a family of eleven putative NSTs from T. cruzi (Table 1). These putative transporters show an overall structural similarity with other members of the NST family. Syntenic homologues to the TcCLB.511301.50 and TcCLB.506509.40 genes are present in Leishmania spp. (L. donovani, L. infantum, L. major, L. mexicana) but not in T. brucei. For all the other putative T. cruzi NSTs, there is a clear syntenic homologue in T. brucei. As has been noted by Capul et al. [12], an interesting role for transporters shared by Leishmania spp and T. cruzi involves UDP-galactofuranose (UDP-Galf), which is present in both trypanosomatids but not in T. brucei. According to our phylogenetic analyses and the TriTrypDB, two T. cruzi NSTs, TcCLB.510355.220 and TcCLB.506793.40, have no clear homologues in T. brucei or Leishmania. Similarly, putative roles for these transporters could be the transport of UDP-xylose (UDP-Xyl) and UDP-rhamnopyranose (UDPRha), which are synthesized only by T. cruzi [26].

Eight nucleotide sugars have been identified in T. cruzi by spectrometry [26], GDP-Man, UDP-GlcNAc, UDPglucose (UDP-Glc), UDP-Gal, UDP-Galf, UDP-Xyl, UDPRha and GDP-fucose. Accordingly, the corresponding sugars have been identified in T. cruzi glycoconjugates [27]. Because we have identified eleven putative NSTs, and assuming that they are all functional, redundancy likely exists between these transporters, as has been reported in other organisms. In fact, the four recently characterized T. brucei NSTs can transport UDP-GlcNAc, two of them (TbNST12) transport UDP-Gal, and the other two (TbNST3-4) transport GDP-Man.

TcNST1 showed the greatest values of similarity (using Blastp and considering Query Coverage $>90 \%$ ) to the $T$. brucei TbNST4 transporter (79 \%), the Arabidopsis thaliana UDP-Gal transporter UDP-GalT1 (51 \%) and the Aspergillus fumigatus UDP-galactofuranose transporter GlfB (44\%). The $K$. lactis UDP-GlcNAc transporter, whose deficient activity in the yeast mutant strain 


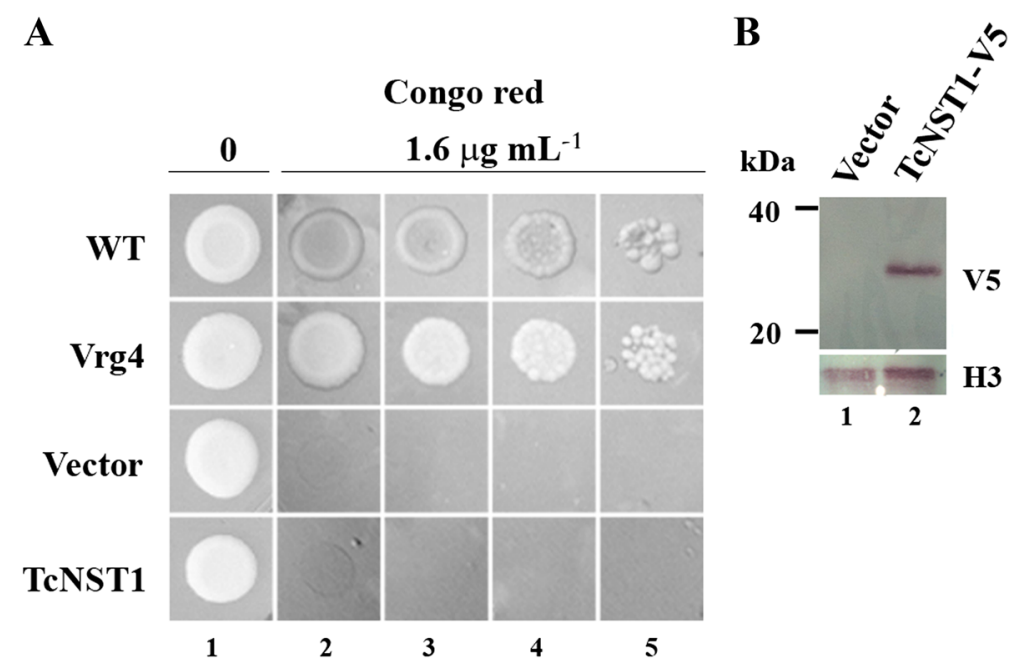

Fig. $\mathbf{3}$ TCNST1 cannot complement a S. cerevisiae mutant strain deficient in GDP-Man transport. a S. cerevisiae strain NDY5 was transfected with TCNST1-V5, the S. cerevisiae GDP-Man Vrg4p transporter (positive control) or empty vector (pYEST-DEST 52, negative control). Wild-type S. cerevisiae (JPY25 6C) cells were included in the analysis for comparison. Transfected cells were grown to exponential phase in liquid cultures of SCM-URA, adjusted to an OD of approximately one (lanes 1 and 2) and submitted to ten-fold serial dilution (lanes 3-5). Cells were then inoculated on solid agar plates containing YP plus $2 \%$ galactose and $1.6 \mu \mathrm{g} / \mathrm{ml}$ Congo red. Growth was assessed after 4 days at $30{ }^{\circ} \mathrm{C}$. $\mathbf{b}$ Western blot analysis of strain NDY5 transfected with empty vector (lane 1) or TCNST1-V5 (lane 2). TCNST1 expression was detected with a mouse anti-V5 antibody. Expression of histone 3 was used as an internal control
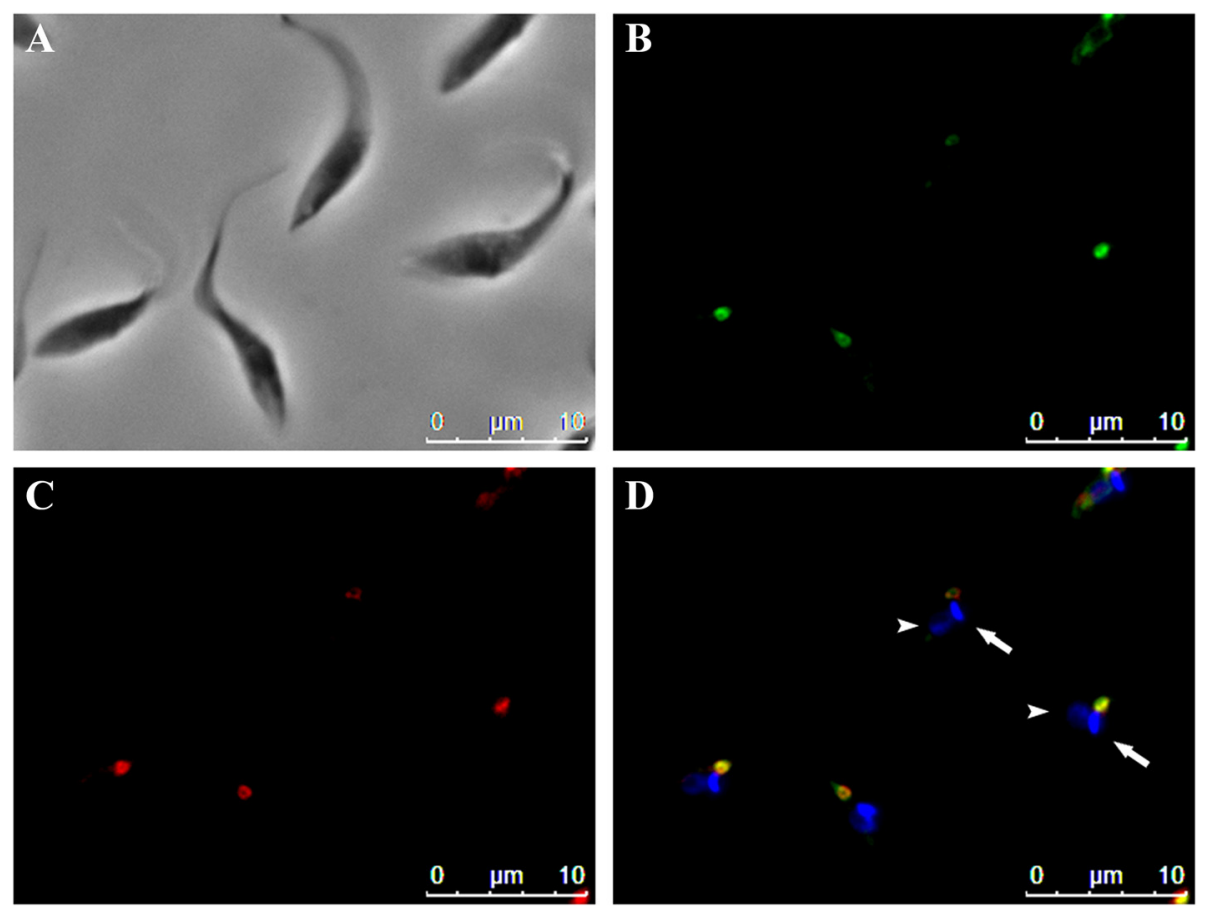

Fig. 4 TCNST1 is localized to the Golgi apparatus. T. cruzi epimastigotes were transfected with a fusion construct in which TcNST1 was tagged at the amino terminus with GFP (GFP-TCNST1). Tagged TCNST1 was localized by fluorescence microscopy after staining with anti-GFP (4b, green) and anti-TcHIP, a Golgi marker (4c, red). Kinetoplasts (large arrow) and nuclei (arrowhead) were stained with DAPI (4d, blue). Secondary antibody controls showed no fluorescence signal (data not shown) 


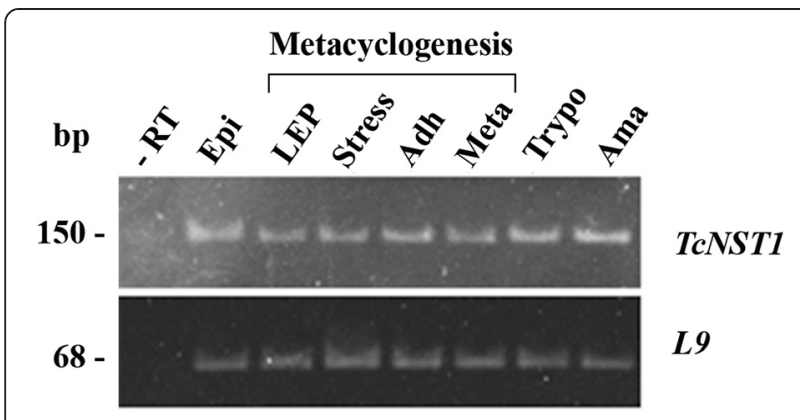

Fig. 5 TcNST1 is expressed at the mRNA level in all life forms of T. cruzi and during metacyclogenesis in vitro. RT-PCR analysis of TCNST1 mRNA expression in epimastigotes (Epi), cell-culture-derived trypomastigotes (Trypo), amastigotes (Ama), metacyclics (Meta) and intermediary forms during metacyclogenesis: epimastigotes in the late exponential phase (LEP), epimastigotes under nutritional stress (Stress) and adhered epimastigotes (Adh). Expression of the 605 ribosomal protein L9 was used as an internal control (TCCLB.504181.10). Aliquots of all samples were pooled together and subjected to reverse transcription without reverse transcriptase $(-\mathrm{RT})$

was compensated by TcNST1, is a more distantly related transporter (Fig. 1), constituting another example of the difficulty involved in determining the substrate specificity of NSTs based on primary sequence analysis.

We also tested the capacity of TcNST1 to transport GDP-Man in yeast cells. The complementation test was negative (Fig. 3). TcNST1 was shown to be expressed in the yeast cells by western blotting, but we cannot rule out that the transporter is not functional in S. cerevisiae by mislocalization. Therefore, even though our results suggest that GDP-Man is not a substrate for TcNST1 in the parasite it is not a definite conclusion. A conserved GALNK motif associated with binding to GDP-Man was identified in the S. cerevisiae VRG4 GDP-Man transporter [28]. The same motif is present in the GDP-Man transporters of Cryptococcus neoformans (GMT1), T. brucei (TbNST3) and L. major (LPG2). Interestingly, in TbNST4, which transports GDP-Man, and TcNST1, this motif is less conserved, but a leucine residue, which is important for VRG4 function at physiological levels [28], is present in TbNST4 (Leu267) but not TcNST1.

NSTs are mainly localized to the Golgi apparatus. However, in trypanosomatids, nucleotide sugars are supposed to be synthesized in glycosomes [29, 30]. It was important, then, to investigate the subcellular localization of TcNST1. Our results (Fig. 4) suggest a clear Golgi localization with no indication of the presence of the transporter in glycosomes.

UDP-GlcNAc is an important substrate for the three trypanosomatids discussed here because it is required for the synthesis of both the conserved core of GPI anchors and the oligosaccharide core of N-glycans [27]. In T. brucei, in which the synthesis of UDP-GlcNAc is essential for parasite survival, the pool of UDP-GlcNAc inside the Golgi lumen is necessary for the synthesis of $N$-acetyllactosamine [31]. In T. cruzi, the Golgi content of UDP-GlcNAc is important for the synthesis of O-linked glycans in mucins, which starts with the attachment of GlcNAc, from UDP-GlcNAc, to a Ser or Thr residue via an $\alpha-N$-acetylglucosaminyltransferase [32-34]. Therefore, the transport of UDP-GlcNAc across the Golgi membrane is essential for the proper synthesis of T. cruzi mucins.

\section{Conclusions}

TcNST1 is a UDP-GlcNAc transporter specifically localized to the Golgi apparatus. Due to its specificity and subcellular localization, it is tempting to speculate that TcNST1 may have a role on the synthesis of $T$. cruzi mucins. Moreover, the expression of TcNST1, at the mRNA level, in all life forms of $T$. cruzi is in agreement with the expression of mucins throughout the life cycle of the parasite. Loss-offunction studies of this transporter are underway to unravel its role in $T$. cruzi glycosylation.

\section{Methods}

\section{Trypanosome cultures}

Epimastigotes of Trypanosoma cruzi Dm28c clone were maintained in liver-infusion tryptose (LIT) medium containing $10 \%$ of fetal bovine serum (FBS) at $28{ }^{\circ} \mathrm{C}$ as previously described [35]. Transfection of epimastigotes in exponential growth phase was performed by electroporation [36] and transfectants were selected in the presence of G418 $(500 \mu \mathrm{g} / \mathrm{ml})$. Metacyclics were obtained by in vitro differentiation of epimastigotes under nutritional stress using chemically defined axenic conditions as described [25]. After differentiation, metacyclic trypomastigotes were purified with ion exchange chromatography on diethylaminoethyl - cellulose columns. The metacyclics obtained after purification usually contain less than $1 \%$ of epimastigotes-like forms. Cell-derived amastigotes and trypomastigotes were obtained by infection of VERO cells with metacyclic forms. Trypomastigotes were released in the supernatant of VERO cell cultures 4 days after infection and harvested by centrifugation. Amastigotes were obtained by disruption of VERO cells 2 days after infection. According to optical microscopic analyses crosscontamination between amastigotes and trypomastigotes are typically less than $5 \%$.

\section{Yeast strains and growth conditions}

The Kluyveromyces lactis wild-type strain MG1/2 and mutant strain KL3 [19] were kindly provided by Dr. C.B. Hirschberg and Dr. C. Bendixen. The Saccharomyces cerevisiae strain JPY25 $6 \mathrm{c}$ and the mutant strain NDY5 were kindly provided by Dr. N. Dean. Yeast strains were grown at $30{ }^{\circ} \mathrm{C}$ on rich medium (YPD) or on synthetic complete medium lacking uracil (YNB supplemented with drop-out medium without uracil, SCM-URA). 


\section{Plasmid construction}

For the complementation assays in K. lactis, the coding sequences of the candidate genes were first amplified by PCR from T. cruzi genomic DNA using specific primers and then cloned into the pE4 vector [20]. The K. lactis UDP-GlcNAc transporter was used as a positive control. The corresponding gene of the wild-type MG1/2 strain was amplified from genomic DNA and cloned into the same vector. The primers and restriction sizes used for cloning are indicated in Additional file 4.

The plasmids used for expression in S. cerevisiae and T. cruzi were constructed using Gateway Technology (Invitrogen). TcNST1 and the S. cerevisiae GDP-Man transporter ScVRG4 were first cloned into the pDONR 221 vector and then transferred by recombination to the destination vectors pYES-DEST 52 (Invitrogen) and pTcGFPN [37] for expression in S. cerevisiae and T. cruzi, respectively. The pYES-DEST 52 vector contains the V5 epitope at the carboxyl-terminus for protein detection and the pTcGFPN vector contains GFP at the amino-terminus and neomycin as a selectable marker. GFP was cloned into the vector pTcGW-3xFlag-Neo, a modified vector derived from the cloning system developed by Batista et al. [37] and kindly provided by Dr. Stenio P Fragoso. All plasmids were confirmed by sequencing.

\section{Complementation assays}

$K$. lactis cells transfected with putative $T$. cruzi NSTs cloned into the pE4 vector [20] were grown on selective medium (SCM-URA). Cell-surface labeling with GS-II lectin was performed according to [20]. Lectin binding was analysed by flow cytometry in a FACS Canto II (Becton \& Dickinson). The sensitivity of the S. cerevisiae strain NDY5 to Congo red was assessed by growth on solid agar plates containing YP plus $2 \%$ galactose and $1.6 \mu \mathrm{g} / \mathrm{ml}$ of the dye. Galactose was used as a carbon source because the pYES-DEST 52 vector contains the yeast GAL1 promoter. Transfected cells in exponential growth were adjusted to an OD of approximately one and then submitted to ten-fold serial dilution. Growth was assessed after 4 days at $30{ }^{\circ} \mathrm{C}$.

\section{Immunofluorescence analysis}

Transfected epimastigotes expressing TcNST1 in fusion with GFP were used for subcellular localization experiments. GFP intrinsic fluorescence of epimastigotes expressing GFP-TcNST1 or GFP-Flag was visualized in live parasites. For co-localization experiments with TcHIP, cells were incubated with rabbit anti-GFP (1:400) and mouse anti-TcHIP (1:100) [24], after fixation (4\% formaldehyde), permeabilization (0.1\% Triton X-100) and blocking (2\% BSA). Secondary antibodies conjugated to Alexa 488 (goat anti-rabbit IgG) and Alexa 594 (goat anti-mouse IgG) were used. Genomic and kinetoplast
DNA was visualized with 4',6-diamidino-2-phenylindole (DAPI). Slides were examined using a Leica DMI6000 B inverted microscope.

\section{Western blot analysis}

Total protein extracts were prepared from exponential growing yeast cells on SCM-URA supplemented with $2 \%$ galactose. Cells were disrupted by glass beads in the presence of RIPA buffer. Cell lysates from $T$. cruzi epimastigotes $\left(5 \times 10^{6}\right.$ parasites) were obtained by direct lysis in Laemmli buffer. After electrophoresis on SDS-PAGE gels (13\%), proteins were electrotransferred onto Optitran BA-S85 membranes (GE Healthcare). Membrane-bound proteins were incubated either with mouse anti-V5 antibody and rabbit anti-human histone 3 or with rabbit anti-GFP and mouse anti-GAPDH. Protein detection was performed using alkaline phosphatase or the Li-COR Odyssey imaging system.

\section{Total RNA isolation and reverse transcription}

Total RNA was isolated from trypanosome cells using the RNeasy ${ }^{\circ}$ Mini Kit (Qiagen). Any contaminant DNA was digested with $1 \mathrm{U}$ per $\mu \mathrm{g}$ of RNA with RNase-free DNase (Promega). Aliquots of $1 \mu \mathrm{g}$ of total RNA were then reverse transcribed to first-strand cDNA using oligo (dT) primers. Amplification by PCR was performed with the primers Tc150F4 (5'GTACTTCAAGACGGCGTTGG3') and Tc150R4 (5'GGCTGACAGTCCGTTCATCT3') for TcCLB.511517.150 and L9F (5'CCTTCACTGCCGTTCG TTGGTTTG3') and L9R (5'ATGCGAGAGTGCCGTG TTGATGGT3') for the 60S ribosomal L9 gene used as an internal control.

\section{Additional files}

Additional file 1: $T$ cruzi candidate genes tested for UDP-GICNAC transport by in vivo complementation assays. K. lactis mutant (KI3) cells were transfected with TCCLB.504057.120 (TCCLB120), TCCLB.504085.60 (TCCLB60) and TCCLB.511277.400 (TCCLB400), the K. lactis UDP-GICNAC transporter (KI UGT, positive control) or empty vector ( $\mathrm{pE} 4$, negative control). Cells were grown as described in the Methods section. After labeling with GS-II lectin (Alexa Fluor 488 conjugate), yeast cells were separated by flow cytometry in a FACS Canto II flow cytometer (Becton \& Dickinson). (TIF $1867 \mathrm{~kb}$ )

Additional file 2: Intrinsic fluorescence emitted by transfected parasites expressing GFP-TcNST1. Epimastigotes expressing the transporter fused to GFP display a single dot at the anterior region of the parasite. Live parasites from cultures of epimastigotes in exponential growth were washed once with PBS, mounted onto slides and immediately visualized in Leica DMI6000 B inverted microscope. (TIF $1386 \mathrm{~kb}$ )

Additional file 3: Expression of GFP-TcNST1 in T. cruzi epimastigotes. Western blot analysis of T. cruzi parasites transfected with GFP-TCNST1 and wild-type cells. Expression of the fusion protein (arrow) was detected with a monoclonal antibody against GFP. Expression of glyceraldehyde 3-phosphate dehydrogenase (GAPDH, $37 \mathrm{kDa}$ ) was used as an internal control. (TIF 69 kb)

Additional file 4: Primers used for cloning of the listed genes in different expression vectors. (DOCX $15 \mathrm{~kb}$ ) 


\section{Abbreviations}

ER: endoplasmic reticulum; GFP: green fluorescence protein; GIPL: glycoinositolphospholipid; GPI: glycosylphosphatidylinositol; GS-II lectin: Griffonia simplicifolia lectin; NST: nucleotide sugar transporter.

\section{Competing interests}

The authors state that they have no competing interests.

\section{Authors' contributions}

CGB and ECR performed most of the experiments. PM carried out metacyclogenesis and gene expression analyses. AK participated in complementation assays and cellular localization experiments. MLZ participated in complementation assays and gene expression analyses. AMA participated in complementation assays. MJS participated in cellular localization experiments. SG helped design the experiments. ASPR supervised the experimental work and drafted the initial manuscript. All authors read and approved the final manuscript.

\section{Acknowledgements}

We are grateful to Dr. Carlos Hirschberg and Dr. Christian Bendixen for providing the K. lactis strains and to Dr. Neta Dean for providing the $S$. cerevisiae strains. We would like to thank Dr. Natalie Cella and Dr. Stenio Fragoso for helpful discussions. In addition, we would like to thank the Program for Technological Development in Tools for Health-PDTIS-FIOCRUZ for the use of its facilities.

This work was supported by Fundação Oswaldo Cruz (Fiocruz), Conselho Nacional de Desenvolvimento e Tecnológico (CNPq) and Fundação Araucária.

\section{Author details}

${ }^{1}$ Present address: Departamento de Parasitologia, Instituto de Ciências Biomédicas, Universidade de São Paulo, São Paulo 05508-000, SP, Brazil. ${ }^{2}$ Present address: Departamento de Imunologia, Instituto de Ciências Biomédicas, Universidade de São Paulo, São Paulo 05508-900, SP, Brazil. ${ }^{3}$ Instituto Carlos Chagas, Fiocruz Paraná, Curitiba 81350-010, PR, Brazil.

\section{Received: 26 June 2015 Accepted: 6 November 2015}

\section{Published online: 21 November 2015}

\section{References}

1. WHO. World Health Organization - WHO. Chagas Disease. Fact sheet N³40. Updated March 2014. Avaiable at http://www.who.int/mediacentre/factsheets/ fs340/en/. 2014.

2. Brener Z. Biology of Trypanosoma cruzi. Annu Rev Microbiol. 1973;27:347-82.

3. Gomes NA, Previato JO, Zingales B, Mendonça-Previato L, DosReis GA. Downregulation of $T$ lymphocyte activation in vitro and in vivo induced by glycoinositolphospholipids from Trypanosoma cruzi. Assignment of the T cellsuppressive determinant to the ceramide domain. J Immunol. 1996;156:628-35.

4. Nogueira NF, Gonzalez MS, Gomes JE, de Souza W, Garcia ES, Azambuja P, et al. Trypanosoma cruzi: involvement of glycoinositolphospholipids in the attachment to the luminal midgut surface of Rhodnius prolixus. Exp Parasitol. 2007; 116:120-8.

5. Mortara RA, da Silva S, Araguth MF, Blanco SA, Yoshida N. Polymorphism of the 35- and 50-kilodalton surface glycoconjugates of Trypanosoma cruzi metacyclic trypomastigotes. Infect Immun. 1992;60:4673-8.

6. Rde Ruiz C, Rigoni VL, Gonzalez J, Yoshida N. The 35/50 kDa surface antigen of Trypanosoma cruzi metacyclic trypomastigotes, an adhesion molecule involved in host cell invasion. Parasite Immunol. 1993;15:121-5.

7. Previato JO, Jones C, Gonçalves LP, Wait R, Travassos LR, Mendonça-Previato L. O-glycosidically linked $\mathrm{N}$-acetylglucosamine-bound oligosaccharides from glycoproteins of Trypanosoma cruzi. Biochem J. 1994;301(Pt 1):151-9.

8. Berninsone PM, Hirschberg CB. Nucleotide sugar transporters of the Golgi apparatus. Curr Opin Struct Biol. 2000;10:542-7.

9. Eckhardt M, Gotza B, Gerardy-Schahn R. Membrane topology of the mammalian CMP-sialic acid transporter. J Biol Chem. 1999;274:8779-87.

10. Liu $L, X u Y X$, Hirschberg CB. The role of nucleotide sugar transporters in development of eukaryotes. Semin Cell Dev Biol. 2010;21:600-8.

11. Ma D, Russell DG, Beverley SM, Turco SJ. Golgi GDP-mannose uptake requires Leishmania LPG2. A member of a eukaryotic family of putative nucleotide-sugar transporters. J Biol Chem. 1997:272:3799-805.

12. Capul AA, Barron T, Dobson DE, Turco SJ, Beverley SM. Two functionally divergent UDP-Gal nucleotide sugar transporters participate in phosphoglycan synthesis in Leishmania major. J Biol Chem. 2007;282:14006-17.
13. Liu L, Xu YX, Caradonna KL, Kruzel EK, Burleigh BA, Bangs JD, et al. Inhibition of nucleotide sugar transport in Trypanosoma brucei alters surface glycosylation. J Biol Chem. 2013;288:10599-615.

14. Caffaro CE, Koshy AA, Liu L, Zeiner GM, Hirschberg CB, Boothroyd JC. A nucleotide sugar transporter involved in glycosylation of the toxoplasma tissue cyst wall is required for efficient persistence of bradyzoites. PLoS Pathog. 2013;9:e1003331.

15. Spath GF, Lye LF, Segawa H, Sacks DL, Turco SJ, Beverley SM. Persistence without pathology in phosphoglycan-deficient Leishmania major. Science. 2003;301:1241-3.

16. Logan-Klumpler FJ, De Silva N, Boehme U, Rogers MB, Velarde G, McQuillan JA, et al. GeneDB-an annotation database for pathogens. Nucleic Acids Res. 2012:40:D98-108.

17. El-Sayed NM, Myler PJ, Bartholomeu DC, Nilsson D, Aggarwal G, Tran AN, et al. The genome sequence of Trypanosoma cruzi, etiologic agent of Chagas disease. Science. 2005;309:409-15.

18. Franzen $\mathrm{O}$, Ochaya S, Sherwood E, Lewis MD, Llewellyn MS, Miles MA, et al, Shotgun sequencing analysis of Trypanosoma cruzi I Sylvio $\times 10 / 1$ and comparison with T. cruzi VI CL Brener. PLoS Negl Trop Dis. 2011:5:e984.

19. Abeijon C, Mandon EC, Robbins PW, Hirschberg CB. A mutant yeast deficient in Golgi transport of uridine diphosphate N-acetylglucosamine. J Biol Chem. 1996;271:8851-4.

20. Guillen E, Abeijon C, Hirschberg CB. Mammalian Golgi apparatus UDP-Nacetylglucosamine transporter: molecular cloning by phenotypic correction of a yeast mutant. Proc Natl Acad Sci U S A. 1998;95:7888-92.

21. Caffaro CE, Luhn K, Bakker H, Vestweber D, Samuelson J, Berninsone P, et al. A single Caenorhabditis elegans Golgi apparatus-type transporter of UDP-glucose, UDP-galactose, UDP-N-acetylglucosamine, and UDP-Nacetylgalactosamine. Biochemistry. 2008;47:4337-44.

22. Caffaro CE, Hirschberg CB, Berninsone PM. Independent and simultaneous translocation of two substrates by a nucleotide sugar transporter. Proc Natl Acad Sci U S A. 2006;103:16176-81.

23. Poster JB, Dean N. The yeast VRG4 gene is required for normal Golgi functions and defines a new family of related genes. J Biol Chem. 1996;271:3837-45.

24. Batista CM, Kalb LC, Moreira CM, Batista GT, Eger I, Soares MJ. Identification and subcellular localization of TCHIP, a putative Golgi zDHHC palmitoyl transferase of Trypanosoma cruzi. Exp Parasitol. 2013;134:52-60.

25. Contreras VT, Salles JM, Thomas N, Morel CM, Goldenberg S. In vitro differentiation of Trypanosoma cruzi under chemically defined conditions. Mol Biochem Parasitol. 1985;16:315-27.

26. Turnock DC, Ferguson MA. Sugar nucleotide pools of Trypanosoma brucei, Trypanosoma cruzi, and Leishmania major. Eukaryot Cell. 2007;6:1450-63.

27. De Lederkremer RM, Agusti R. Glycobiology of Trypanosoma cruzi. Adv Carbohydr Chem Biochem. 2009;62:311-66.

28. Gao XD, Nishikawa A, Dean N. Identification of a conserved motif in the yeast golgi GDP-mannose transporter required for binding to nucleotide sugar. J Biol Chem. 2001;276:4424-32.

29. Roper JR, Ferguson MA. Cloning and characterisation of the UDP-glucose 4'-epimerase of Trypanosoma cruzi. Mol Biochem Parasitol. 2003;132:47-53.

30. Mariño K, Güther MLS, Wernimont AK, Qiu W, Hui R, Ferguson MAJ. Characterization, localization, essentiality, and high-resolution crystal structure of glucosamine 6-phosphate $\mathrm{N}$-acetyltransferase from Trypanosoma brucei. Eukaryot Cell. 2011;10:985-97.

31. Stokes MJ, Guther ML, Turnock DC, Prescott AR, Martin KL, Alphey MS, et al. The synthesis of UDP-N-acetylglucosamine is essential for bloodstream form trypanosoma brucei in vitro and in vivo and UDP-N-acetylglucosamine starvation reveals a hierarchy in parasite protein glycosylation. J Biol Chem. 2008:283:16147-61

32. Previato JO, Sola-Penna M, Agrellos OA, Jones C, Oeltmann T, Travassos LR et al. Biosynthesis of O-N-acetylglucosamine-linked glycans in Trypanosoma cruzi. Characterization of the novel uridine diphospho-N-acetylglucosamine: polypeptide $\mathrm{N}$-acetylglucosaminyltransferase-catalyzing formation of N-acetylglucosamine alpha1-O-threonine. J Biol Chem. 1998;273:14982-8.

33. Heise $N$, Singh $D$, van der Wel H, Sassi SO, Johnson JM, Feasley $C L$, et al. Molecular analysis of a UDP-GlcNAc:polypeptide alpha-Nacetylglucosaminyltransferase implicated in the initiation of mucin-type O-glycosylation in Trypanosoma cruzi. Glycobiology. 2009;19:918-33.

34. Koeller CM, van der Wel H, Feasley $\mathrm{CL}$, Abreu F, da Rocha JDB, Montalvão F, et al. Golgi UDP-GIcNAc:polypeptide O-a-N-Acetyl-d-glucosaminyltransferase 2 (TcOGNT2) regulates trypomastigote production and function in Trypanosoma cruzi. Eukaryot Cell. 2014;13:1312-27. 
35. Camargo EP. Growth and differentiation in Trypanosoma cruzi. I. Origin of metacyclic trypanosomes in liquid media. Rev Inst Med Trop Sao Paulo. 1964;6:93-100

36. Lu HY, Buck GA. Expression of an exogenous gene in Trypanosoma cruzi epimastigotes. Mol Biochem Parasitol. 1991;44:109-14.

37. Batista M, Marchini FK, Celedon PA, Fragoso SP, Probst CM, Preti $\mathrm{H}$, et al. A high-throughput cloning system for reverse genetics in Trypanosoma cruzi. BMC Microbiol. 2010;10:259.

Submit your next manuscript to BioMed Central and take full advantage of:

- Convenient online submission

- Thorough peer review

- No space constraints or color figure charges

- Immediate publication on acceptance

- Inclusion in PubMed, CAS, Scopus and Google Scholar

- Research which is freely available for redistribution 